\title{
TU/e EmonOWEN

\section{Carrier frequency offset estimation for multi-user MIMO OFDM uplink using CAZAC sequences}

\section{Citation for published version (APA):}

Wu, Y., Attallah, S., \& Bergmans, J. W. M. (2009). Carrier frequency offset estimation for multi-user MIMO OFDM uplink using CAZAC sequences. In Proceedings of the IEEE Wireless Communications and Networking Conference (WCNC), 5-9 May 2009, Budapest, Hungary (pp. 1-5). Institute of Electrical and Electronics Engineers. https://doi.org/10.1109/WCNC.2009.4917620

DOI:

10.1109/WCNC.2009.4917620

Document status and date:

Published: 01/01/2009

\section{Document Version:}

Publisher's PDF, also known as Version of Record (includes final page, issue and volume numbers)

\section{Please check the document version of this publication:}

- A submitted manuscript is the version of the article upon submission and before peer-review. There can be important differences between the submitted version and the official published version of record. People interested in the research are advised to contact the author for the final version of the publication, or visit the $\mathrm{DOI}$ to the publisher's website.

- The final author version and the galley proof are versions of the publication after peer review.

- The final published version features the final layout of the paper including the volume, issue and page numbers.

Link to publication

\section{General rights}

Copyright and moral rights for the publications made accessible in the public portal are retained by the authors and/or other copyright owners and it is a condition of accessing publications that users recognise and abide by the legal requirements associated with these rights.

- Users may download and print one copy of any publication from the public portal for the purpose of private study or research.

- You may not further distribute the material or use it for any profit-making activity or commercial gain

- You may freely distribute the URL identifying the publication in the public portal.

If the publication is distributed under the terms of Article 25fa of the Dutch Copyright Act, indicated by the "Taverne" license above, please follow below link for the End User Agreement:

www.tue.nl/taverne

Take down policy

If you believe that this document breaches copyright please contact us at:

openaccess@tue.nl

providing details and we will investigate your claim. 


\title{
Carrier Frequency Offset Estimation for Multi-User MIMO OFDM Uplink Using CAZAC Sequences
}

\author{
Yan $\mathrm{Wu}^{*}$, Samir Attallah ${ }^{\dagger}$, J.W.M. Bergmans * \\ *Department of Electrical Engineering \\ Eindhoven University of Technology (TU/e), The Netherlands \\ ${ }^{\dagger}$ School of Science and Technology, SIM University (SIM), Singapore
}

\begin{abstract}
In the uplink of multi-user multiple input multiple output (MIMO) orthogonal frequency division multiplexing (OFDM) systems, there are multiple carrier frequency offsets (CFO's) from the multiple users. In this paper, we study algorithms to estimate these multiple CFO values. We first derive the maximum likelihood (ML) estimator and show that the complexity of the ML estimator increases exponentially with the number of users so that the estimator is not suitable for practical implementations. To reduce the complexity, we propose a suboptimal algorithm using constant amplitude zero autocorrelation (CAZAC) sequences. The complexity of the proposed method increases only linearly with the number of users. Using computer simulations, we compare the performance using CAZAC training sequences with that using the $m$ sequence and the short training field (STF) of the IEEE 802.11n systems. The results show that in the low to medium SNR regions, the performance using CAZAC sequences is very close to the single-user Cramer-Rao bound. For high SNR regions, an error floor exists due to multiple access interference (MAI). The error floor using CAZAC sequences is more than 10 times smaller compared to the error floor using the other two sequences.
\end{abstract}

\section{INTRODUCTION}

The multiple input multiple output (MIMO) system increases the information capacity of rich scattering wireless fading channels enormously by employing multiple antennas at both the transmitter and the receiver [1] [2]. Combining MIMO with orthogonal frequency division multiplexing (OFDM) enables efficient implementation of the MIMO concept for frequency selective fading channels. An extension of the MIMO-OFDM system is the multi-user MIMO-OFDM system illustrated in Figure 1. In such a system, multiple users, each with one or multiple antennas, transmit simultaneously using the same spectrum. The receiver is a base station equipped with multiple antennas. It uses spatial processing techniques to separate the signals from different users. This system is also known as the virtual MIMO system [3].

Carrier frequency offset (CFO) is caused by the doppler effect of the channel and the difference between the transmitter and receiver local oscillators (LOs). In OFDM systems, CFO destroys the orthogonality between the subcarriers and causes inter-carrier interference (ICI). Therefore, to ensure reliable performance of OFDM systems, the CFO must be accurately

This research was supported in part by the Dutch Min. Econ. Affairs under the IOP-Gen Com program (IGC0502)

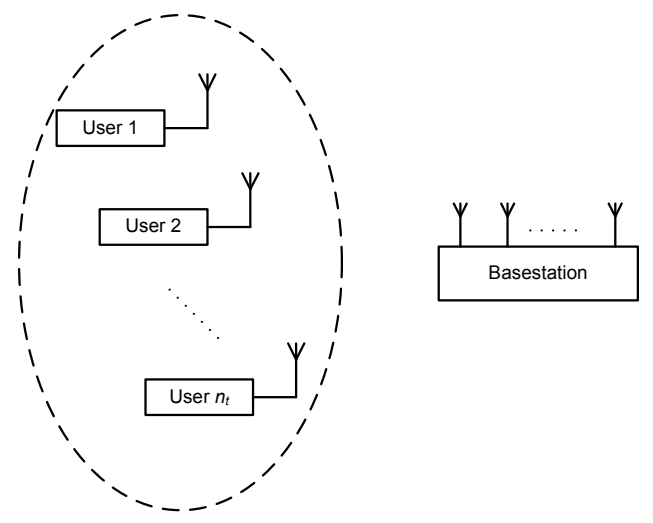

Fig. 1. Illustration of multi-user MIMO OFDM systems.

estimated and compensated. For single input single output (SISO) OFDM systems, periodic training sequences are used in [4] and [5] to estimate the CFO. It is shown that such CFO estimator reaches the Cramer-Rao bound (CRB) with low computational complexity. Similar idea was extended to collocated MIMO-OFDM systems [6] [7] [8], where all the transmit antennas are driven by a centralized LO and so are all the receive antennas. In this case, the CFO is still a single parameter. The CFO estimation is more complicated for a multi-user MIMO-OFDM system. In this system, each user has its own LO, while the multiple antennas at the basestation (receiver) is driven by a common LO. Therefore, in the uplink, the receiver needs to estimate multiple $\mathrm{CFO}$ values for all the users. The CFO estimation for multi-user MIMO systems in flat fading channels was studied in [9] and [10]. In [11], a semi-blind method was proposed to estimate the frequency offset and channel coefficients for the uplink of multi-user MIMO-OFDM systems in frequency selective fading channels.

In this paper, we study the CFO estimation in the uplink of multi-user MIMO-OFDM systems using training sequences. We first derive the maximum likelihood (ML) estimator for the multiple $\mathrm{CFO}$ values. To obtain the ML estimates requires a search over all the possible CFO values. The complexity of such a search grows exponentially with the number of users and is prohibitive for practical implementations. To reduce the complexity, we propose a sub-optimal method using 
constant amplitude zero autocorrelation (CAZAC) training sequences, which have zero autocorrelation for any nonzero circular shifts. Using the proposed method, the CFO estimates can be obtained using simple correlation operations and the complexity of this method grows only linearly with the number of users. We show that the multiple CFO values destroy the orthogonality between the training sequences from different users. This introduces multiple access interference (MAI). We derive an expression for the signal to interference ratio (SIR) and show that it is dependent of the CFO values and the power delay profiles (PDP) of the channel. We use computer simulations to study the performance of the proposed method. The results show that for low to medium signal to noise ratio (SNR), the proposed method performs close to the singleuser CRB. An error floor exists for high SNR, which is due to MAI. We compare the performance using the CAZAC sequences with the performance using IEEE 802.11n short training field (STF) [12] and the $\mathrm{m}$ sequences, which are two other sequences with good autocorrelation properties. The results show that the error floor using the CAZAC sequences is more than 10 times smaller compared to the other two sequences.

The rest of the paper is organized as follows. In Section II, we present the system model and derive the ML estimator for the multiple CFO values. The sub-optimal CFO estimation algorithm using CAZAC sequences is proposed in Section III. The performance of the proposed method was evaluated using computer simulations in Section IV and Section V concludes the paper.

\section{SySTEM MODEL}

In this paper, we study a multi-user MIMO-OFDM system with $n_{t}$ users, where each user has a single transmit antenna. The basestation has $n_{r}$ receive antennas, with $n_{r} \geq n_{t}$, The received signal at the $i$ th receive antenna can be written as

$$
r_{i}(k)=\sum_{m=1}^{n_{t}} e^{j \phi_{m} k} \sum_{d=0}^{L-1} h_{i, m}(d) s_{m}(k-d)+n_{i}(k),
$$

where $\phi_{m}$ is the CFO value for the $m$ th user, $k$ is the timing index, and $L$ is the number of multi-path components in the channel. From (1), we can see that we have $n_{t}$ different CFO values $\left(\phi_{m}\right.$ 's) to estimate. The received signal in (1) can be written in equivalent matrix form as

$$
\mathbf{r}_{i}=\sum_{m=1}^{n_{t}} \mathbf{E}\left(\phi_{m}\right) \mathbf{S}_{m} \mathbf{h}_{i, m}+\mathbf{n}_{i},
$$

where $\mathbf{r}_{i}=\left[r_{i}(0), \cdots, r_{i}(L-1)\right]^{T}$ and superscript $T$ denotes transpose. The CFO matrix of user $m$ is denoted as $\mathbf{E}\left(\phi_{m}\right)$, which is a diagonal matrix with diagonal elements equal to $\left[1, \exp \left(j \phi_{m}\right), \cdots, \exp \left(j(L-1) \phi_{m}\right)\right]$. We use $\mathbf{S}_{m}$ to denote the transmitted signal matrix for the $m$ th user, which is a circulant matrix with the first row defined by $\left[s_{m}(0), s_{m}(L-\right.$ $\left.1), s_{m}(L-2), \cdots, s_{m}(1)\right]$. The channel impulse response vector between the $m$ th user and the $i$ th receive antenna is denoted as $\mathbf{h}_{i, m}$. Collecting the received signals from all receive antennas for $N$ samples, we have

$$
\mathcal{R}=\mathcal{A}(\Phi) \mathcal{H}+\mathcal{N}
$$

where

$$
\begin{gathered}
\mathcal{R}=\left[\mathbf{r}_{1} \cdots, \mathbf{r}_{n_{r}}\right]_{N \times n_{r}} \\
\mathcal{A}(\Phi)=\left[\mathbf{E}\left(\phi_{1}\right) \mathbf{S}_{1}, \cdots, \mathbf{E}\left(\phi_{n_{t}}\right) \mathbf{S}_{n_{t}}\right]_{N \times\left(N \times n_{t}\right)} .
\end{gathered}
$$

For easy understanding, we use a subscript under the square bracket to denote the matrix size. The vector $\Phi=$ $\left[\phi_{1}, \cdots, \phi_{n_{t}}\right]$ is the CFO vector containing the CFO values from all users, and the channels of all users are stacked into the channel matrix $\mathcal{H}$ given as

$$
\mathcal{H}=\left[\begin{array}{c}
\mathcal{H}_{1} \\
\vdots \\
\mathcal{H}_{n_{t}}
\end{array}\right]_{\left(N \times n_{t}\right) \times n_{r}},
$$

with $\mathcal{H}_{i}=\left[\mathbf{h}_{1, i}, \cdots, \mathbf{h}_{n_{r}, i}\right]_{N \times n_{r}}$ being he channel matrix for the $i$ th user. Here we assume that $N \geq L$ and that $\mathbf{h}_{i, m}$ is an $N \times 1$ vector by appending the original $L \times 1$ vector $\mathbf{h}_{i, m}$ with $N-L$ zeros. The noise matrix is given by $\mathcal{N}=\left[\mathbf{n}_{1}, \cdots, \mathbf{n}_{n_{r}}\right]$.

Because the noise is Gaussian and uncorrelated, the likelihood function for the channel $\mathcal{H}$ and CFO values $\Phi$ can be written as

$$
\Lambda(\mathcal{H}, \Phi)=\frac{1}{\left(\pi \sigma_{n}^{2}\right)^{N \times n_{r}}} \exp \left\{-\frac{1}{\sigma_{n}^{2}}\|\mathcal{R}-\mathcal{A}(\Phi) \mathcal{H}\|^{2}\right\} .
$$

Following a similar approach in [13], we find that for a fixed CFO vector $\Phi$, the ML estimate of the channel is given by

$$
\mathcal{H}(\Phi)=\left[\mathcal{A}^{H}(\Phi) \mathcal{A}(\Phi)\right]^{-1} \mathcal{A}^{H}(\Phi) \mathcal{R}
$$

Substituting (5) into (4) and after some algebraic manipulations, we obtain the ML estimate of the CFO vector $\Phi$ given by

$$
\hat{\Phi}=\arg \max _{\Phi}\left\{\operatorname{tr}\left(\mathcal{R}^{H} \mathcal{B R}\right)\right\},
$$

where

$$
\mathcal{B}=\mathcal{A}(\Phi)\left[\mathcal{A}^{H}(\Phi) \mathcal{A}(\Phi)\right]^{-1} \mathcal{A}^{H}(\Phi),
$$

and $\operatorname{tr}(\bullet)$ denotes the trace of a matrix. To obtain the ML estimate of the CFO vector $\Phi$, a search needs to be performed over the possible CFO values from all the users. The complexity of this search increases exponentially with the number of users and this search is not practical.

\section{CAZAC SEQUENCES FOR THE CFO ESTIMATION IN MULTI-USER MIMO-OFDM SYSTEMS}

To reduce the complexity of the CFO estimation for multiuser MIMO-OFDM systems, in this section, we propose a suboptimal algorithm making use of CAZAC sequences. CAZAC sequences are special sequences with constant amplitude elements and zero autocorrelation for any non-zero circular shifts. This means for a length- $N$ CAZAC sequence, we have $s(n)=\exp \left(j \theta_{n}\right)$ and

$$
R(k)=\sum_{n=1}^{N} s(n) s^{*}(n \ominus k)=\left\{\begin{array}{cc}
N & k=0 ; \\
0 & k \neq 0 .
\end{array}\right.
$$


for all values of $k=0,1, \cdots, N-1$. Here we use $\ominus$ to denote circular subtraction. Let $\mathbf{S}$ be a circulant matrix with the first row equal to $[s(1), s(N), s(N-1), \cdots, s(2)]$. The autocorrelation property of CAZAC sequences can be written in equivalent matrix form as

$$
\mathbf{S}^{H} \mathbf{S}=N \mathbf{I},
$$

where $\mathbf{I}$ is the identity matrix. This means that $\mathbf{S}$ is both a unitary and a circulant matrix. Examples of CAZAC sequences include the Frank-Zadoff sequence [14] and the Chu sequence [15].

In [16], we showed that for collocated MIMO-OFDM systems, using CAZAC training sequences results in efficient joint $\mathrm{CFO}$ and channel estimation. The CFO estimation also achieves CRB performance. Here, we extend the idea to the CFO estimation in multi-user MIMO-OFDM systems. Let the training sequence of the first user be $\mathbf{s}_{1}$. The training sequence of the $m$ th user is the circularly shifted version of the 1st user, i.e. $s_{m}(n)=s_{1}\left(n \ominus \tau_{m}\right)$, where $\tau_{m}$ denotes the shift value. It is straight forward to show that the training sequences between different users have the following properties

- The autocorrelation of the circulant matrix for the $i$ th user satisfies

$$
\mathbf{S}_{i}^{H} \mathbf{S}_{i}=N \mathbf{I}
$$

for $i=1, \cdots, n_{t}$.

- The cross correlation satisfies

$$
\mathbf{S}_{i}^{H} \mathbf{S}_{j}=\mathfrak{I}^{i-j}
$$

where $\mathfrak{I}^{i-j}$ denotes a matrix which results from circularly shifting the one elements of the identify matrix to the right by $\tau_{j}-\tau_{i}$.

For easy CFO estimation, two periods of the training sequence are used as in SISO-OFDM systems [5]. In this case, the received signals in the two periods can be written as

$\mathcal{R}=\left[\begin{array}{ccc}\mathbf{E}\left(\phi_{1}\right) \mathbf{S}_{1} & \cdots & \mathbf{E}\left(\phi_{n_{t}}\right) \mathbf{S}_{n_{t}} \\ e^{j N \phi_{1}} \mathbf{E}\left(\phi_{1}\right) \mathbf{S}_{1} & \cdots & e^{j N \phi_{n_{t}}} \mathbf{E}\left(\phi_{n_{t}}\right) \mathbf{S}_{n_{t}}\end{array}\right] \mathcal{H}+\mathcal{N}$

Without loss of generality, we show how to estimate the CFO of the 1st user and the same procedure can be applied to all the other users. Because the same procedure is applied to all the users, the complexity of such a CFO estimation method increases linearly with the number of users.

We first consider a special case when all the other users are synchronized except for the first user, i.e. $\phi_{m}=0$ for $m=2, \cdots, n_{t}$. We cross correlate the training sequence of the first user with the received signal and we get

$$
\begin{aligned}
\tilde{\mathcal{Y}}_{1} & =\mathbf{W}_{1} \mathcal{R} \\
& =\left[\begin{array}{cc}
\mathbf{S}_{1}^{H} & \mathbf{0} \\
\mathbf{0} & \mathbf{S}_{1}^{H}
\end{array}\right]\left[\begin{array}{ccc}
\mathbf{E}\left(\phi_{1}\right) \mathbf{S}_{1} & \cdots & \mathbf{S}_{n_{t}} \\
e^{j N \phi_{1}} \mathbf{E}\left(\phi_{1}\right) \mathbf{S}_{1} & \cdots & \mathbf{S}_{n_{t}}
\end{array}\right] \mathcal{H}+\mathcal{N}^{\prime} \\
& =\left[\begin{array}{c}
\mathbf{S}_{1}^{H} \mathbf{E}\left(\phi_{1}\right) \mathbf{S}_{1} \mathcal{H}_{1}+\sum_{m t}^{n_{t}} \mathbf{S}_{1}^{H} \mathbf{S}_{m} \mathcal{H}_{m} \\
e^{j N \phi_{1}} \mathbf{S}_{1}^{H} \mathbf{E}\left(\phi_{1}\right) \mathbf{S}_{1} \mathcal{H}_{1}+\sum_{m=2}^{n_{t}} \mathbf{S}_{1}^{H} \mathbf{S}_{m} \mathcal{H}_{m}
\end{array}\right]+\mathcal{N}^{\prime} \\
& =\left[\begin{array}{c}
\mathbf{S}_{1}^{H} \mathbf{E}\left(\phi_{1}\right) \mathbf{S}_{1} \mathcal{H}_{1}+\sum_{m=2}^{n_{t}} \mathfrak{I}^{\tau_{m}} \mathcal{H}_{m} \\
e^{j N \phi_{1}} \mathbf{S}_{1}^{H} \mathbf{E}\left(\phi_{1}\right) \mathbf{S}_{1} \mathcal{H}_{1}+\sum_{m=2}^{n_{t}} \mathfrak{I}^{\tau_{m}} \mathcal{H}_{m}
\end{array}\right]+\mathcal{N}^{\prime},(12)
\end{aligned}
$$

where $\mathfrak{I}^{\tau_{m}}$ is an matrix resulting from circularly shifting the identity matrix to the right by $\tau_{m}$ samples. Therefore, $\mathfrak{I}^{\tau_{m}} \mathcal{H}_{m}$ produces a matrix resulting by circularly shifting the rows of $\mathcal{H}_{m} \tau_{m}$ samples downwards.

We make sure that the circular shift between the $m-1$ th and $m$ th users are larger than length of the channel impulse response, i.e. $\tau_{m}-\tau_{m-1} \geq L$. Since the channel order is $L$, only the first $L$ rows in the $N \times n_{r}$ matrix $\mathcal{H}_{m}$ are nonzero. Therefore, the first $L$ rows of $\mathfrak{I}^{\tau_{m}} \mathcal{H}_{m}$ are all zero for $m=$ $2, \cdots, n_{t}$. As a result, the first $L$ rows of $\mathcal{Y}_{1}$ are free of the interference from all the other users. Let us define $\mathcal{I}_{L}$ as the first $L$ rows of the $N \times N$ identity matrix, we have

$\mathcal{Y}_{1}=\left[\begin{array}{cc}\mathcal{I}_{L} & \mathbf{0} \\ \mathbf{0} & \mathcal{I}_{L}\end{array}\right] \tilde{\mathcal{Y}}_{1}=\left[\begin{array}{c}\mathcal{I}_{L} \mathbf{S}_{1}^{H} \mathbf{E}\left(\phi_{1}\right) \mathbf{S}_{1} \mathcal{H}_{1} \\ e^{j N \phi_{1}} \mathcal{I}_{L} \mathbf{S}_{1}^{H} \mathbf{E}\left(\phi_{1}\right) \mathbf{S}_{1} \mathcal{H}_{1}\end{array}\right]+\mathcal{N}^{\prime \prime}$

We can see that the purpose of $\mathcal{I}_{L}$ is to select the first $L$ rows from the matrix $\mathbf{S}_{1}^{H} \mathbf{E}\left(\phi_{1}\right) \mathbf{S}_{1} \mathcal{H}_{1}$. Because the CFO's of all the other users are 0 , the shift orthogonality between the training sequences is maintained. In this case, $\mathcal{Y}_{1}$ is free of interference from the other users. Following a similar approach as in [16], we can show that the ML estimate of the CFO for the first user is given by

$$
\hat{\phi}_{1}=\frac{1}{N} \angle\left\{\sum_{k=1}^{L} \sum_{m=1}^{n_{r}} \mathcal{Y}_{1}^{*}(k, m) \mathcal{Y}_{1}(k+N, m)\right\},
$$

and this can be easily implemented using correlations. Notice that to ensure $\tau_{m}-\tau_{m-1} \geq L$ for all $m$, we need to have the training sequence length $N \geq n_{t} L$.

When the other users' CFO values are not zero, $\mathcal{Y}_{1}$ is given by

$$
\begin{aligned}
\mathcal{Y}_{1} & =\left[\begin{array}{c}
\mathcal{I}_{L} \mathbf{S}_{1}^{H} \mathbf{E}\left(\phi_{1}\right) \mathbf{S}_{1} \mathcal{H}_{1} \\
e^{j N \phi_{1}} \mathcal{I}_{L} \mathbf{S}_{1}^{H} \mathbf{E}\left(\phi_{1}\right) \mathbf{S}_{1} \mathcal{H}_{1}
\end{array}\right] \\
+ & {\left[\begin{array}{c}
\mathcal{I}_{L} \sum_{m}^{n_{t}} \mathbf{S}_{1}^{H} \mathbf{E}\left(\phi_{m}\right) \mathbf{S}_{m} \mathcal{H}_{m} \\
\mathcal{I}_{L} \sum_{m=2}^{n_{t}} e^{j N \phi_{m}} \mathbf{S}_{1}^{H} \mathbf{E}\left(\phi_{m}\right) \mathbf{S}_{m} \mathcal{H}_{m}
\end{array}\right]+\mathcal{N}^{\prime \prime} } \\
& =\left[\begin{array}{c}
\mathcal{I}_{L} \mathbf{S}_{1}^{H} \mathbf{E}\left(\phi_{1}\right) \mathbf{S}_{1} \mathcal{H}_{1} \\
e^{j N \phi_{1}} \mathcal{I}_{L} \mathbf{S}_{1}^{H} \mathbf{E}\left(\phi_{1}\right) \mathbf{S}_{1} \mathcal{H}_{1}
\end{array}\right]+\mathcal{V}+\mathcal{N}^{\prime \prime}
\end{aligned}
$$

From (15), we can see that the orthogonality between the training sequences from different users is destroyed by the presence of the CFO's $\phi_{m}$. As a result, there is an extra MAI term $\mathcal{V}$ in the received signal. This interference is independent of the noise and hence it will cause an irreducible error floor in performance of the CFO estimator given in (14). The covariance matrix of the MAI term can be expressed as in (16) on top of the next page. Here, we assume the channels between different transmit and receive antennas are uncorrelated in space. We also assume different paths in the multi-path channel are also uncorrelated. If we let $\mathbf{p}_{i, m}=$ $\left[p_{i, m}(1), \cdots, p_{i, m}(L), 0, \cdots, 0\right]^{T}$ be a $N \times 1$ vector containing the $L \times 1$ PDP of the channel between the $m$ th user and the $i$ th receive antenna and $(N-L) \times 1$ zero vector, we have

$$
E\left\{\mathcal{H}_{m} \mathcal{H}_{n}^{H}\right\}=\left\{\begin{array}{cc}
\operatorname{diag}(\mathbf{0}) & m \neq n \\
\operatorname{diag}\left(\sum_{i=1}^{n_{r}} \mathbf{p}_{i, m}\right) & n=m
\end{array}\right.
$$




$$
\begin{aligned}
& E\left\{\mathcal{V V}^{H}\right\}=E\left\{\left[\begin{array}{c}
\mathcal{I}_{L} \sum_{m=2}^{n_{t}} \mathbf{S}_{1}^{H} \mathbf{E}\left(\phi_{m}\right) \mathbf{S}_{m} \mathcal{H}_{m} \\
\mathcal{I}_{L} \sum_{m=2}^{n_{t}} e^{j N \phi_{m}} \mathbf{S}_{1}^{H} \mathbf{E}\left(\phi_{m}\right) \mathbf{S}_{m} \mathcal{H}_{m}
\end{array}\right]\right. \\
& \left.\left[\begin{array}{ll}
\sum_{m=2}^{n_{t}} \mathcal{H}_{m}^{H} \mathbf{S}_{m}^{H} \mathbf{E}^{H}\left(\phi_{m}\right) \mathbf{S}_{1} \mathcal{I}_{L}^{H} \quad \sum_{m=2}^{n_{t}} \mathcal{H}_{m}^{H} e^{-j N \phi_{m}} \mathbf{S}_{m}^{H} \mathbf{E}^{H}\left(\phi_{m}\right) \mathbf{S}_{1} \mathcal{I}_{L}^{H}
\end{array}\right]\right\}
\end{aligned}
$$

Defining $\mathbf{P}_{m}=\operatorname{diag}\left(\sum_{i=1}^{n_{r}} \mathbf{p}_{i, m}\right)$, the signal to interference ratio (SIR) of the $m$ th user is given by

$\operatorname{SIR}_{m}=\frac{\operatorname{trace}\left[\mathcal{I}_{L}\left\{\mathbf{S}_{m}^{H} \mathbf{E}\left(\phi_{m}\right) \mathbf{S}_{m} \mathbf{P}_{m} \mathbf{S}_{m}^{H} \mathbf{E}^{H}\left(\phi_{m}\right) \mathbf{S}_{m}\right\} \mathcal{I}_{L}^{H}\right]}{\operatorname{trace}\left[\mathcal{I}_{L}\left\{\sum_{k=1, k \neq m}^{n_{t}} \mathbf{S}_{m}^{H} \mathbf{E}\left(\phi_{k}\right) \mathbf{S}_{k} \mathbf{P}_{k} \mathbf{S}_{k}^{H} \mathbf{E}^{H}\left(\phi_{k}\right) \mathbf{S}_{m}\right\} \mathcal{I}_{L}^{H}\right]}$

We can see that the SIR is a function of the CFO values for all the users and the PDP of the channels.

\section{Simulation Results}

In this section, we present the simulation results of the proposed CFO estimation algorithm using CAZAC sequences. We simulate a system with two users and each user has one transmit antenna. The basestation has two receive antennas. The OFDM system has 128 subcarriers with length 16 cyclic prefix. We compare the performance of CFO estimation using CAZAC sequences with the following two sequences which also have good autocorrelation properties

1) IEEE $802.11 \mathrm{n}$ short training field [12];

2) $m$ sequences.

In the simulations, we use the $802.11 \mathrm{n}$ STF for $40 \mathrm{MHz}$ operations which has a length of 32 . For the $\mathrm{m}$ sequence, we use a sequence length of 31 . To provide a fair comparison, we compare the performance using the 802.11n STF with a length-32 Chu (CAZAC) sequence generated by [15]

$$
s(n)=\exp \left[j \pi \frac{(n-1)^{2}}{N}\right],
$$

and we compare the performance with the $\mathrm{m}$ sequence using a length-31 Chu sequence generated by [15]

$$
s(n)=\exp \left[j \pi \frac{(n-1) n}{N}\right] .
$$

We provide comparisons for multipath channels with uniform and exponential PDP. For channels with exponential PDP , the root mean square (rms) delay spread is equal to 1 sample period. The CFO is normalized with respect to the subcarrier spacing. The actual CFO values for the two users are modeled as random variables uniformly distributed between $[-0.5,0.5]$. The mean square error (MSE) of the CFO estimation is defined by

$$
\text { MSE }=\frac{1}{N_{s}} \sum_{i=1}^{N_{s}}\left(\frac{\hat{\phi}_{i}-\phi_{0}}{2 \pi / K}\right)^{2},
$$

where $\hat{\phi}_{i}$ and $\phi_{0}$ represent the estimated and true CFO's, respectively, $K$ is the number of subcarriers, and $N_{s}$ denotes the total number of Monte Carlo trials.

The performance of CFO estimation using the 802.11n STF and $N=32 \mathrm{Chu}$ sequence is shown in Figure 2 and Figure 3 for channels with uniform and exponential PDP. Here we

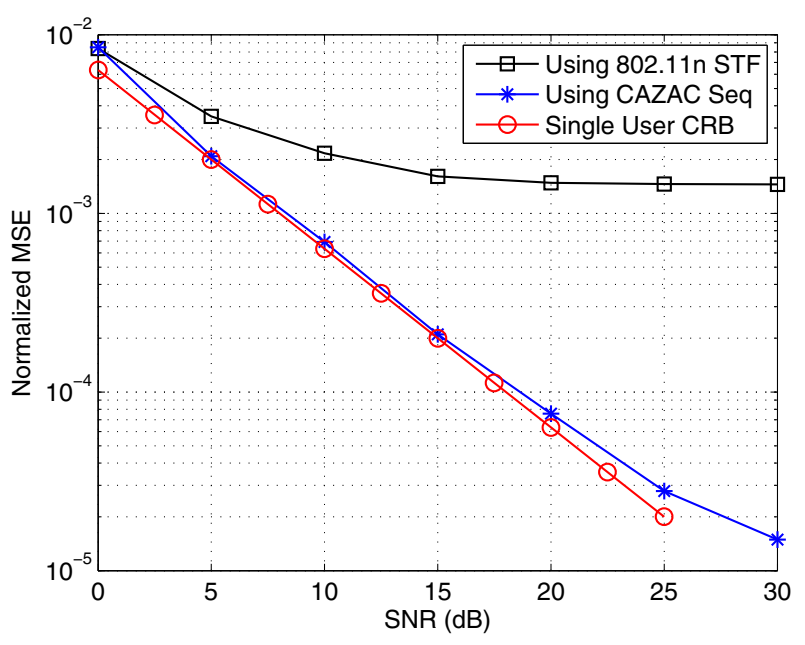

Fig. 2. MSE of CFO estimation using $N=32$ CAZAC sequences for uniform power delay profile.

use 16-tap multipath channels and the circular shift between the training sequences of the two users $\tau_{2}=16$. To gauge the performance of the CFO estimation, we also included the single-user CRB in the comparison. The single-user CRB is obtained by assuming no MAI and can be shown to be [17]

$$
\mathrm{CRB}=\frac{K^{2}}{4 \pi^{2} n_{r} N^{3} \gamma}
$$

where $\gamma$ is the SNR per receive antenna. From the results, we can see that the CFO estimation using the 802.11n STF has a very high error floor above MSE of $10^{-3}$. The performance using CAZAC sequences is much better. In low to medium SNR regions, the performance is very close to the single-user CRB. Error floor starts to appear at SNR of about $25 \mathrm{~dB}$. The error floor is around 100 times smaller compared to the error floor using the 802.11n STF.

The performance of the CFO estimation using the $N=31$ $\mathrm{m}$ sequence and Chu sequence is shown in Figure 4 and Figure 5 for channels with uniform and exponential PDP. Here to satisfy the condition of $N \geq n_{t} L$, we use 15-tap multipath fading channels and the circular shift between user 1 and 2 's training sequence is also set to 15 . Again using CAZAC sequences leads to a much better performance. We can see that in low to medium SNR regions, their performance is very close to the single-user CRB. The error floor using CAZAC sequences is more than 10 times smaller than that using the $m$ sequence. 


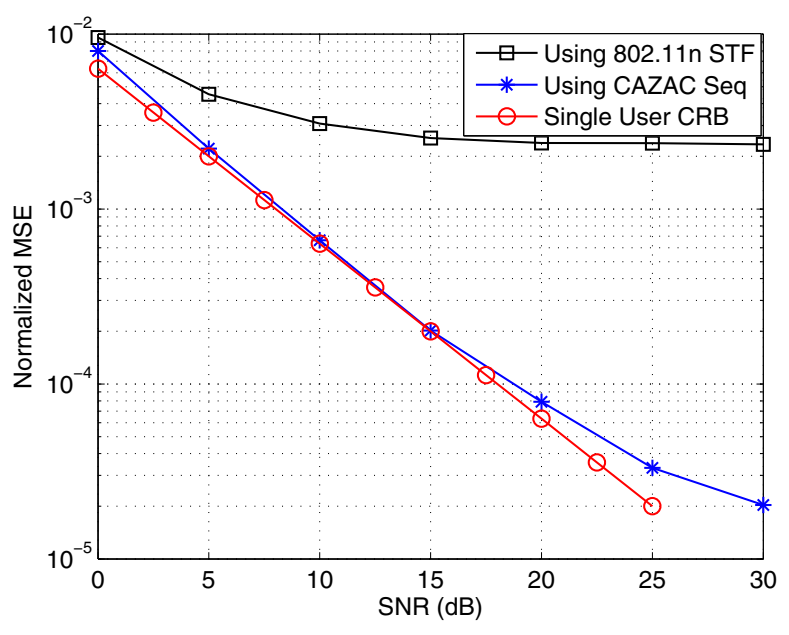

Fig. 3. MSE of CFO estimation using $N=32$ CAZAC sequences for exponential power delay profile with rms delay spread of 1.

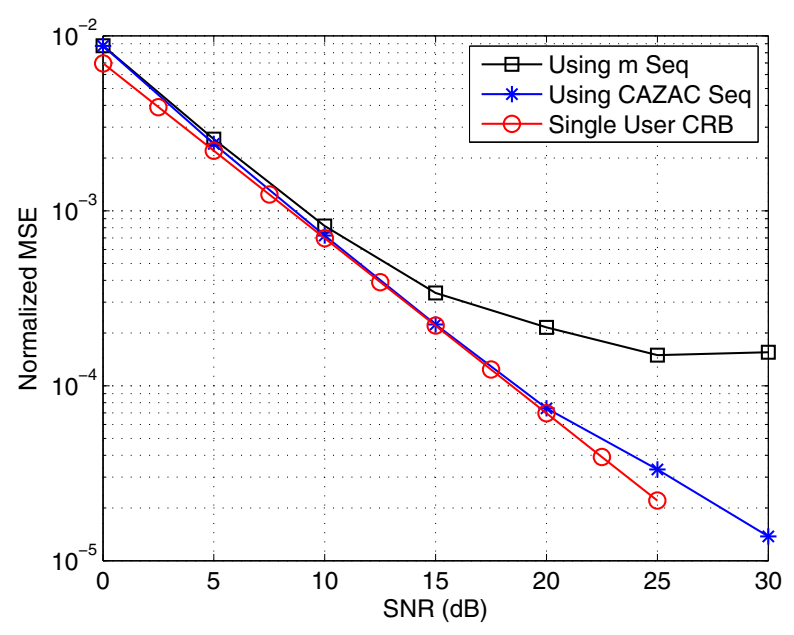

Fig. 4. Comparison of $\mathrm{CFO}$ estimation using $N=31$ CAZAC sequences and $\mathrm{m}$ sequence for uniform power delay profile.

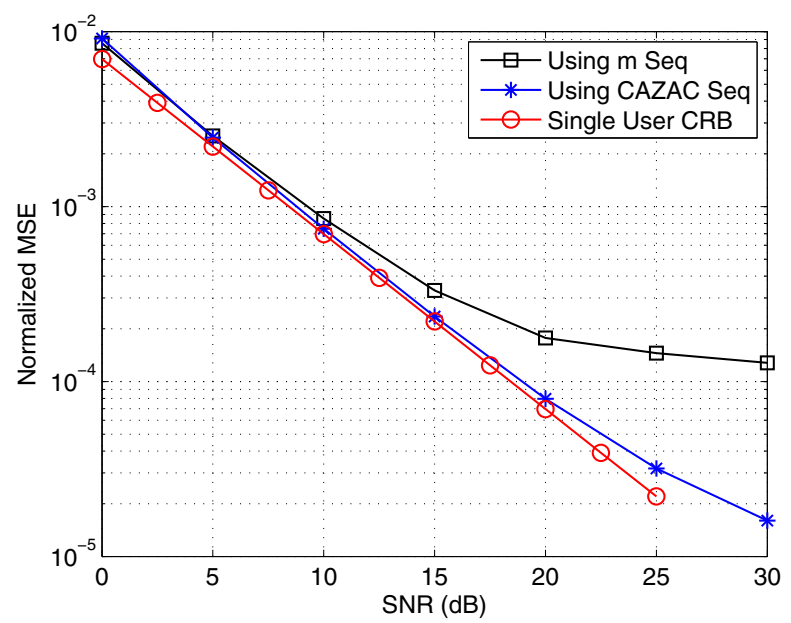

Fig. 5. Comparison of CFO estimation using $N=31$ CAZAC sequences and $\mathrm{m}$ sequence for exponential power delay profile with rms delay spread of 1.

\section{CONCLUSIONS}

In this paper, we studied the CFO estimation problem for the uplink of the multi-user MIMO-OFDM systems. We showed that the maximum likelihood estimator has a complexity that increases exponentially with the number of users. To reduce the complexity, we proposed a suboptimal method using CAZAC sequences. The complexity of the proposed method increases only linearly with the number of users. Computer simulations showed that the proposed method performs close to the single-user Cramer-Rao bound in low to medium SNR regions. For high SNR, the method has an error floor due to the multiple access interference caused by the multiple CFO's. However, the error floor using the CAZAC sequences is more than 10 times smaller than using other practical sequences such as the IEEE $802.11 \mathrm{n}$ short training field and the $\mathrm{m}$ sequence.

\section{REFERENCES}

[1] G. J. Foschini, "Layered space-time architecture for wireless communications in a fading environment when using multi-element antennas," Bell Labs Technical Journal, vol. 1, no. 2, pp. 41-59, Autumn 1996.

[2] I. Telatar, "Capacity of multi-antenna gaussian channels," European Trans. Telecommun. Related Technol., vol. 10, pp. 585-595, Nov-Dec 1999.

[3] M. Dohler, E. Lefranc, and H. Aghvami, "Virtual antenna arrays for future mobile communication systems," in IEEE ICT, Beijing, China, 2002.

[4] P. Moose, "A technique for orthogonal frequency division multiplexing frequency offset correction," IEEE Trans. Commun., vol. 42, no. 10, pp. 2908-2914, Oct 1994.

[5] T. Schmidl and D. Cox, "Robust frequency and timing synchronization for ofdm," IEEE Trans. Commun., vol. 45, no. 12, pp. 1613-1621, Dec 1997.

[6] A. Mody and G. Stüber, "Synchronization for mimo ofdm systems," in Proc. IEEE Global Telecommuncations Conference 2001, vol. 1, Nov 2001, pp. $509-513$.

[7] G. Stuber, J. Barry, S. McLaughlin, Y. Li, M. Ingram, and T. Pratt, "Broadband mimo-ofdm wireless communications," Proc. IEEE, vol. 92, no. 2, pp. 271-294, 2004.

[8] A. Zelst and T. Schenk, "Implementation of a mimo ofdm-based wireless lan system," IEEE Trans. Signal Process., vol. 52, no. 2, pp. 483-494, Feb 2004.

[9] O. Besson and P. Stoica, "On parameter estimation of mimo flat-fading channels with frequency offsets," IEEE Trans. Signal Process., vol. 51, no. 3, pp. 602-613, Mar. 2003.

[10] Y. Yao and T.-S. Ng, "Correlation-based frequency offset estimation in mimo system," in Proc. VTC 2003-Fall Vehicular Technology Conference 2003 IEEE 58th, vol. 1, 2003, pp. 438-442 Vol.1.

[11] Y. Zeng, A. Leyman, and T.-S. Ng, "Joint semiblind frequency offset and channel estimation for multiuser mimo-ofdm uplink," IEEE Trans. Commun., vol. 55, no. 12, pp. 2270-2278, 2007.

[12] IEEE, "IEEE P802.11n/D1.10 wireless LAN medium access control (MAC) and physical layer (PHY) specifications: Enhancements for higher throughput," Feb 2007.

[13] M. Morelli and U. Mengali, "Carrier-frequency estimation for transmissions over selective channles," IEEE Trans. Commun., vol. 48, no. 9, pp. 1580-1589, Sept. 2000.

[14] R. Frank, S. Zadoff, and R. Heimiller, "Phase shift pulse codes with good periodic correlation properties (corresp.)," IRE Transactions on Information Theory, vol. 8, no. 6, pp. 381-382, 1962.

[15] D. Chu, "Polyphase codes with good periodic correlation properties (corresp.)," IEEE Trans. Inf. Theory, vol. 18, no. 4, pp. 531-532, 1972.

[16] Y. Wu, S. Attallah, and J. W. M. Bergmans, "Efficient training sequence for joint carrier frequency offset and channel estimation for mimo-ofdm systems," in Proc. IEEE International Conference on Communications ICC '07, 2007, pp. 2604-2609.

[17] T. Schenk and A. Zelst, "Frequency synchronization for mimo ofdm wireless lan systems," in Proceedings IEEE Vehicular Technology Conference (VTC) 2003 Fall, vol. 2, Oct 2003, pp. 781-785. 